\title{
Michigan Ultra-Cold Polarized Atomic Hydrogen Jet Target
}

\author{
B.B. Blinov ${ }^{1}$, S.E. Gladycheva ${ }^{1}$, T. Kageya ${ }^{1}$, D.Yu. Kantsyrev ${ }^{1}$, \\ A.D. Krisch ${ }^{1}$, V.G. Luppov ${ }^{1}$, V.S. Morozov ${ }^{1}$, J.R. Murray ${ }^{1}$, \\ R.S. Raymond ${ }^{1}$, N.S. Borisov ${ }^{2}$, V.V. Fimushkin ${ }^{2}$, V.N. Grishin ${ }^{3}$, \\ A.I. Mysnik ${ }^{3}$, D. Kleppner ${ }^{4}$ \\ ${ }^{1}$ Randall Lab. of Physics, University of Michigan, Ann Arbor, MI - 48109-1120, USA \\ ${ }^{2}$ Joint Institute for Nuclear Research, RU-141980, Dubna, Russia \\ ${ }^{3}$ Institute for High Energy Physics, RU-142284, Protvino, Russia \\ ${ }^{4}$ Department of Physics, Massachusetts Inst. of Technology, Cambridge, Massachusetts 02139, \\ USA
}

\begin{abstract}
To study spin effects in high energy collisions, we are developing an ultra-cold high-density jet target of proton-spin-polarized hydrogen atoms. The target uses a 12 Tesla magnetic field and a $0.3 \mathrm{~K}$ separation cell coated with superfluid helium- 4 to produce a slow monochromatic electron-spin-polarized atomic hydrogen beam, which is then focused by a superconducting sextupole into the interaction region. In recent tests, we studied a polarized beam of hydrogen atoms focused by the superconducting sextupole into a compression tube detector, which measured the polarized atoms' intensity. The Jet produced, at the detector, a spin-polarized atomic hydrogen beam with a measured intensity of about $2.8 \cdot 10^{15} \mathrm{H} \mathrm{s}^{-1}$ and a FWHM area of less than $0.13 \mathrm{~cm}^{2}$. This intensity corresponds to a free jet density of about $1 \cdot 10^{12} \mathrm{H} \mathrm{cm}^{-3}$ with a proton polarization of about $50 \%$. When the transition RF unit is installed, we expect a proton polarization higher than $90 \%$.
\end{abstract}

To study spin effects in high energy collisions, we are developing an ultra-cold high-density jet target of proton-spin-polarized hydrogen atoms (Michigan Jet). The jet first uses a high magnetic field $(12 \mathrm{~T})$ and an ultra-cold $(0.3 \mathrm{~K})$ separation cell coated with a superfluid helium-4 film to produce a slow monochromatic electron-spin-polarized atomic hydrogen beam; an rf transition unit will then convert this beam into a proton-spin-polarized beam [1].

A layout of the Michigan Jet is shown in Fig. 1. Atomic hydrogen is produced in a room- temperature if dissociator and guided to the ultra-cold separation cell coated with superfluid helium-4 to depress the surface recombination of atoms. The double walls of the cell form the mixing chamber of the dilution refrigerator. The cell's entrance and exit apertures are respectively located at about $95 \%$ and $50 \%$ of the 12 Tesla central magnetic field of the superconducting solenoid. After the hydrogen atoms are thermalized by 


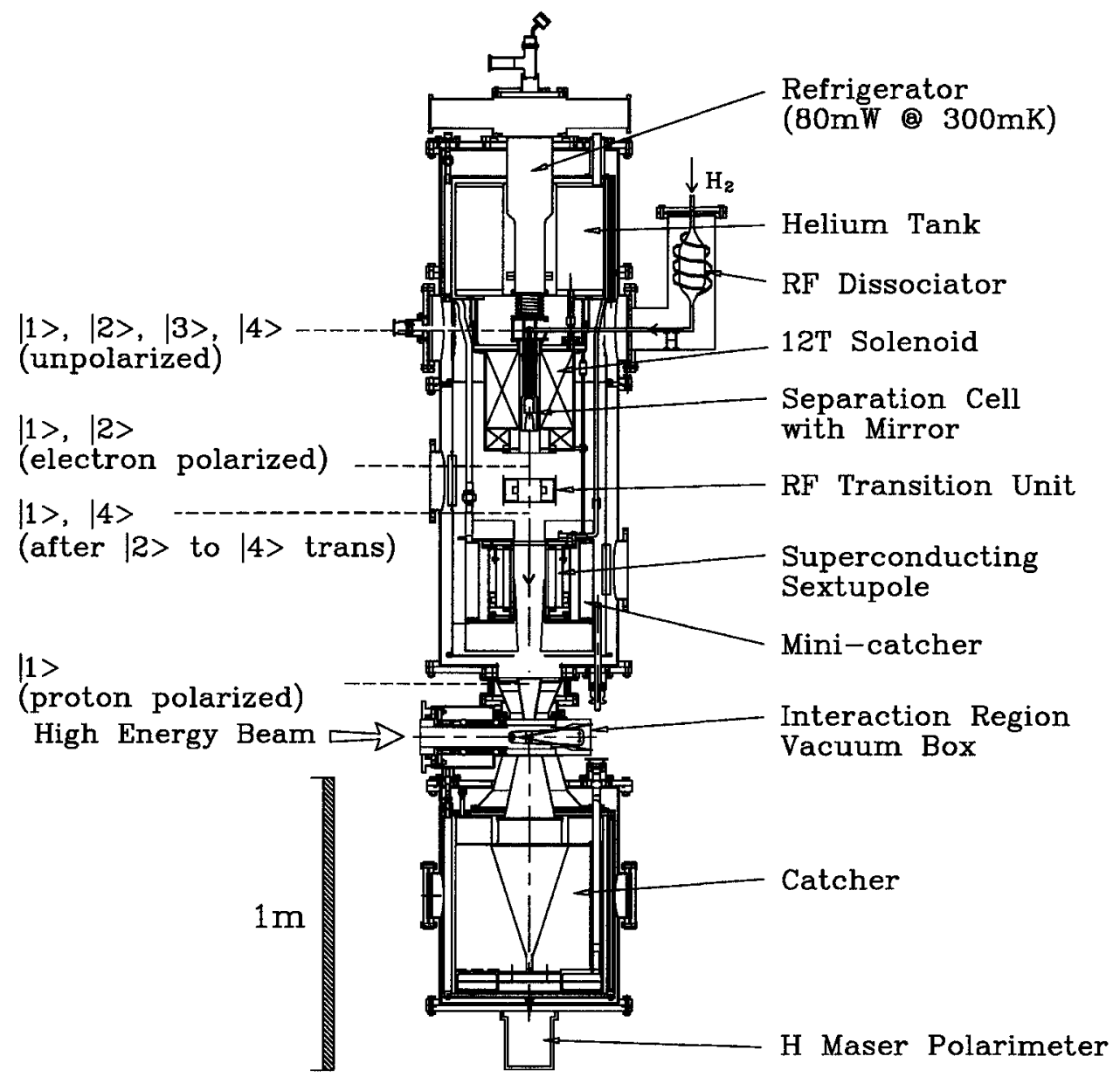

FIGURE 1. The layout of the Michigan ultra-cold jet.

collisions with the cell surface, the magnetic field gradient physically separates the atoms according to their electron-spin states. The atoms in the two lowest hyperfine states 3 and 4 are attracted toward the high field region and escape from the cell. They quickly recombine on bare surfaces and are cryopumped. The atoms in the two higher hyperfine states 1 and 2 are repelled toward the low field region and effuse from the exit aperture, forming an electron-spin polarized beam. To increase the jet density we use a polished gold-coated copper focusing mirror covered with a helium-4 superfluid film similar to the prototype mirror [2]. After an rf transition unit, which interchanges atoms in states 2 and 4, the beam passes through a superconducting sextupole. 
The sextupole selects atoms in electron spin state $+1 / 2$ by focusing atoms in state 1 into the interaction region and defocusing atoms in state 4 , which are then cryopumped. The proton-spin polarized beam then passes through the interaction region and is caught below by a cryopumping catcher. A maser polarimeter below the catcher monitors the beam proton polarization.

Most of the Michigan Jet parts have been fabricated and successfully tested. This hardware includes a 12 Tesla superconducting solenoid, a dilution refrigerator with a cooling power of almost $80 \mathrm{~mW}$ at $300 \mathrm{mK}$, a $20 \mathrm{~cm}$ long superconducting sextupole magnet with iron poles and a $10.5 \mathrm{~cm}$ diameter bore, a cryocondensation pump with a measured pumping speed of about $1.2 \cdot 10^{7} \mathrm{1} / \mathrm{s}$ $\left(4.2 \cdot 10^{26}\right.$ atoms/(Torr s)), and a hydrogen maser polarimeter capable of monitoring the polarization to about $\pm 2 \%$ in a few minutes.

In recent tests we studied a polarized beam of hydrogen atoms focused by the superconducting sextupole into a compression tube detector, which measured the polarized atoms' intensity. The measured compression tube signal versus focusing sextupole current is shown in Fig. 2. As we expected the sextupole curve shape corresponds to calculated beam velocity distribution [3].

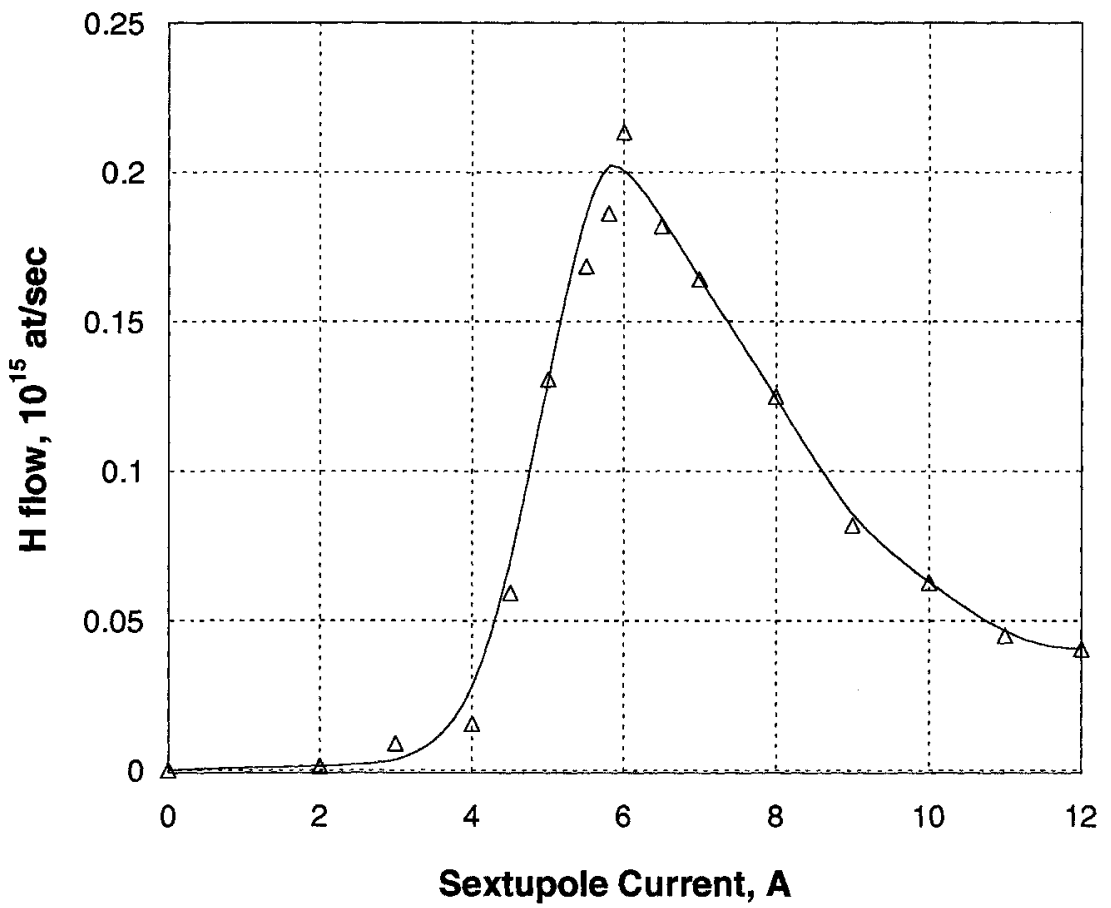

FIGURE 2. The measured compression tube signal versus the sextupole current. The line is a fit to the data . 


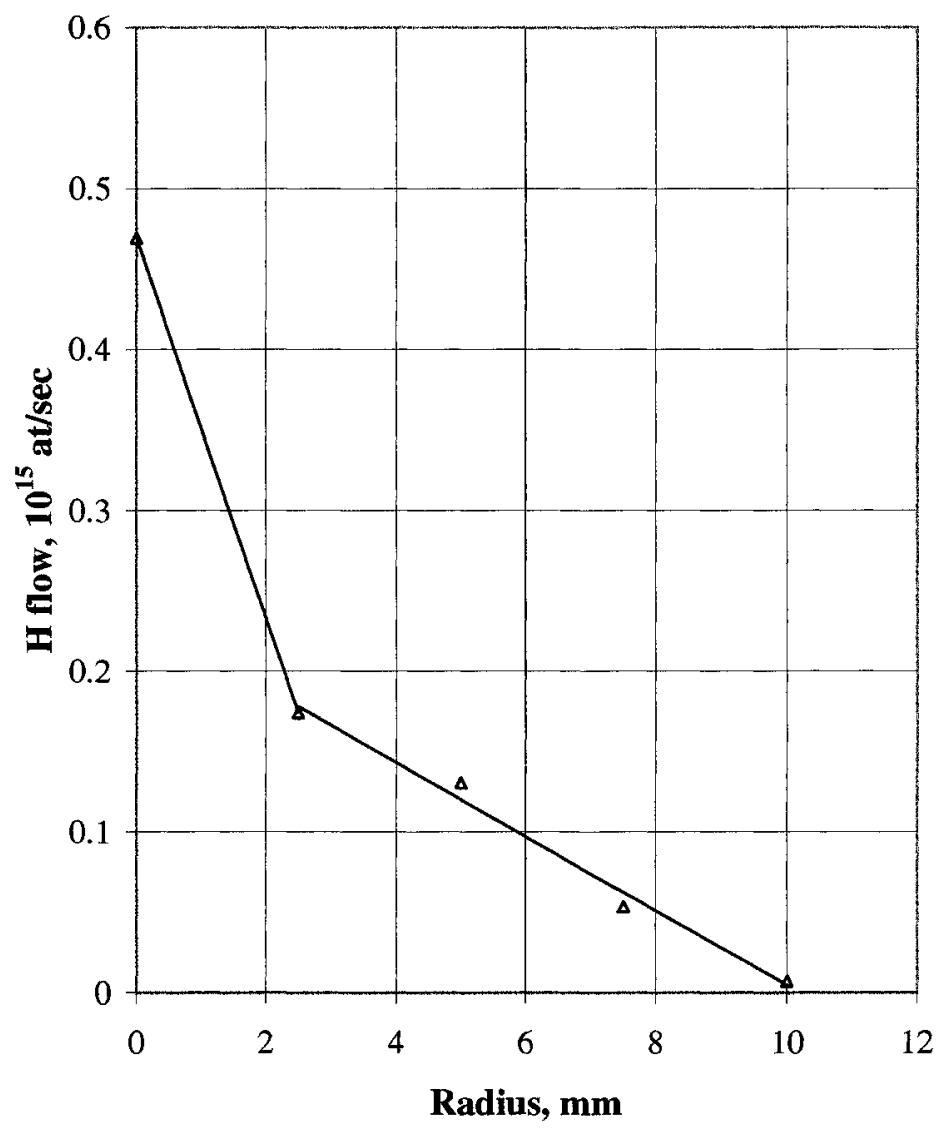

FIGURE 3. The measured radial beam distribution. The line is a fit to the data .

According to our Monte-Carlo simulations [3] the ultra-cold beam is very monochromatic due to the low temperature and the much larger magnetic field gradient acceleration along the solenoid axis. As a result we expected a small size of the focused beam. Indeed, the measured radial beam distribution shown in Fig. 3 gives a FWHM of only about $4 \mathrm{~mm}$. A test of the new superfluid- ${ }^{4} \mathrm{He}-$ coated parabolic mirror, attached to the separation cell, appeared to increase the beam intensity by a factor of about 3 , as expected [4].

The highest measured spin-polarized atomic hydrogen jet intensity to the $12 \mathrm{~mm}$ by $2.5 \mathrm{~mm}$ compression tube slot is about $2.8 \cdot 10^{15} \mathrm{H} \mathrm{s}^{-1}$. Within the $4 \mathrm{~mm}$ FWHM area of $0.13 \mathrm{~cm}^{2}$, this intensity corresponds to a free jet density of $1 \cdot 10^{12} \mathrm{H} \mathrm{cm}^{-3}$. The Jet's total thickness is about $6 \cdot 10^{11} \mathrm{H} \mathrm{cm}^{-2}$. So far, the intensity is limited by the high insulation vacuum pressure due to the evaporation of the separation cell's helium film. 
The electron-spin polarized beam has a proton polarization of about $50 \%$. The electron polarization will be converted into proton polarization by adiabatic passage through an if transition unit with a novel ring dielectric resonator that accepts the $6 \mathrm{~cm}$ beam diameter. A room temperature prototype if unit was tested with a maximum measured transition efficiency of $97 \%$. A preliminary design of the cryogenic rf unit has been fabricated and tested [5].

\section{ACKNOWLEDGEMENTS}

Supported by a research grant from the U.S. Department of Energy.

\section{REFERENCES}

1. V.G. Luppov et al., Status of the Mark-II Polarized Hydrogen Jet Target, in Proceedings of the 13-th International Symposium on High-Energy Spin Physics, Protvino, Russia, September 1998, edited by N.E. Tyurin et al, World Scientific, 1999, pp.409-411.

2. V.G. Luppov, W.A. Kaufman, K.M. Hill, R.S. Raymond, and A.D. Krisch, Phys. Rev. Lett. 71, 2405 (1993).

3. V.G. Luppov et al., Status of the Mark-II Polarized Hydrogen Jet Target, in Proceedings of the 12-th International Symposium on High-Energy Spin Physics, Amsterdam, The Netherlands, September 1996, edited by C.W. de Jager et al, World Scientific, 1997, pp.434-437.

4. T. Kageya, Private communication.

5. R.S. Raymond, Development of a Large-bore Cryogenic 2-4 Transition Unit, in Proceedings of the International Workshop on Polarized Sources and Targets, edited by A. Gute et al., Erlangen, Germany, September 1999. 